\title{
An Introspective Performance Analysis of Threshold-based Segmentation Techniques on Digital Mammograms
}

\author{
A. Nithya ${ }^{1}$, P. Shanmugavadivu ${ }^{2}$ \\ ${ }^{1,2}$ Department of Computer Science and Applications, \\ The Gandhigram Rural Institute (Deemed to be University), Dindigul, Tamil Nadu, India.
}

\begin{abstract}
:
Image segmentation, as a pre-processing step, plays a vital role in medical image analysis. The variants of threshold-based image segmentation methods are proved to offer feasible and optimal solutions to extract the region of interest (RoI), from medical images. Digital mammograms are used as a reliable source of breast cancer prognosis and diagnosis. Thresholding is a simple and effective strategy that finds applications in image processing and analysis. This research aimed to analyze the performance behaviour of a few threshold-based segmentation methods with respect to the intensity distribution of the input mammograms. For this analytical research, six automated thresholding segmentation techniques were chosen: Kapur, Otsu's, Isoentropic, Ridler \& Calvard's, Kittler \& Illingworth's, and Yen. The performance and behaviour of those methods were validated on the digital mammogram images of mini-MIAS database featured with Fatty $(F)$, Fatty-Glandular $(G)$, and Dense-Glandular $(D)$ breast tissues. Those methods were analyzed on two metrics viz., Region Non-Uniformity (RNU) and computation time. The results of this research confirm that Ridler \& Calvard's method gives the best segmentation results for Dense-Glandular, Isoentropic method gives better segmentation results for Fatty and Yen method works well on the Fatty-Glandular normal mammogram images.
\end{abstract}

Keywords: Image Segmentation, Digital Mammogram, Threshold-based segmentation, Breast cancer, Breast Cancer Diagnosis. 


\section{Introduction}

Breast cancer, characterized by the irregular growth of malignant cells in the form of a tumor in the breast tissue, is declared as the second major cause of cancer death among women. Mammography is a low-dose X-ray imaging modality that can visualize the internal anatomy of the breast. Mammography can detect 80-90 percent of asymptomatic breast cancers in women. [1]. Mammography-based screening is considered one of the most common reliable and noninvasive methods for breast cancer detection [2]. Early and accurate detection of breast cancer, based on the masses and/or lesions visualized by the digital mammogram is proved to contain the mortality rate.

Image segmentation is an essential pre-processing step in the computer-aided analysis of mammograms, regardless of the subsequent processing steps. The foreground breast tissue segmented out of the mammogram serves as a crucial input for the successive processing steps, namely image registration, fusion and automated detection of disease. A portion of pectoral muscle visible in mediolateral oblique (MLO) mammograms, helps to determine the boundaries of the region of interest (RoI) and provides vital information about the location and orientation of the breast as well as the overall intensity of the gray levels in the image. [3].

The presence of artifacts in a mammogram challenges the efficacy of the image segmentation algorithms and consequently, abnormal region/object identification as well as disease classification. These artifacts include tags, pectoral muscles, and other non-breast regions. Researchers have developed numerous artefacts removal techniques, which may increase the accuracy of segmentation techniques while extracting the designated regions/tumors in a mammogram. Development of automated/semi-automated computer-aided diagnosis (CAD) systems for breast cancer detection should necessarily possess the computational elements for the removal of noise, artifacts, pectoral muscle, non-breast regions etc. [4].

Thresholding is a popular and traditional principle used in the various phases of digital image processing. It helps to transform the higher intensity image values to distinguish foreground objects from the background, based on an arbitrary or image-dependent value, known as threshold value [5]. The classical types of thresholding are: global and local thresholds. The former one refers to a particular threshold value that is used consistently throughout the image.. The threshold value can be a statistical measure of the input image or be an arbitrary value. The latter one is adaptively chosen based on the statistics of the virtual partitions of the image, referred to as sub- 
images. In certain algorithms, the optimal threshold value is determined by the trial and error method. Threshold-based methods are proved to be computationally simple and thus require less execution time. Due to its generality of computation and its applications, several threshold based segmentation techniques have been developed and are vibrantly used in various medical image processing applications such as digital mammogram analysis for breast cancer detection and brain abnormality detection [6].

Ergin et al., proposed a threshold-based segmentation using median filtering, thresholding and morphological operations. The authors have attempted to remove tiny background artifacts like labels as well as to segment the breast region. The authors have validated the accuracy of segmentation based on the visual perception of the segmented region(s) [7]. Palkar and Agrawal proposed a global threshold-based approach for removing breast boundaries using global threshold value as 32 and median filter. The findings were analysed by visual analysis of the segmentation [8].

Ibrahim et al., developed a method based on fixed threshold value as 18 and its performance is evaluated by human visual perception [9]. For extracting the breast borders, Qayyum and Basit used Otsu's thresholding, in which the images using median filter, prior to thresholding and morphological operations for artifacts removal and segmentation. The breast region is then determined by the entity with the most connected pixels. The authors have validated the accuracy of segmentation based on the visual perception, their proposed approach was able to give quality and appropriate segmentation in $96.89 \%$ and $2.48 \%$ of the images, respectively [10].

Salama et al., developed a system that uses a $5 \times 5$ median filter on the mammogram images before thresholding [11]. Esener et al., developed a similar technique, using adaptive median filtering prior to Otsu's thresholding process [12]. Ancy and Nair proposed a method to perform contrast enhancement using Gamma correction proceeding to thresholding [13].

Bajaj et al., developed a global thresholding-based method for extracting breast boundaries from images, in which the images were segmented using a threshold and then supplemented with morphological operations to remove the background items such as scanning artifacts and labels [14]. Selvathi and Poornila develop a global thresholding method to extract breast boundaries, in which the input images are transformed into binary images based on a threshold value of 18 , to extract the region of interest. After that, the breast boundary was smoothed using morphological operations using a structuring element of a 5 pixel radius. [15]. The review of literature endorses 
the significant role of threshold in the segmentation process. This affirmation motivated the authors to analyse six prominent threshold-based segmentation methods.

The performance of the six most popular threshold-based segmentation methods viz., Isoentropic, Kapur, Kittler \& Illingworth's, Otsu's, Ridler \& Calvard's, and Yen is analyzed in this article. This study aimed to examine the effects of these six techniques on mammogram images to ascertain the accuracy of segmentation, on a variety of mammograms featured with Fatty, FattyGlandular, and Dense-Glandular breast tissues from the mini-MIAS database's normal class. The comparative analysis was performed using the metrics namely, region non-uniformity and computation time.

In this article, the description of the experimental dataset is given in section 2 and section 3 explains the mechanics of experimental analysis; section 4 explains the results and discussion, and the conclusions of this study are furnished in section 5 .

\section{Experimental Dataset}

The images for experimentation were taken from the mini-Mammographic Image Analysis Society (MIAS) database of mammograms [16]. In MLO, there are 322 mammogram images. The actual MIAS database, digitized in the order of 50- $\mu \mathrm{m}$ pixel edge, was reduced to a $200-\mu \mathrm{m}$ pixel edge; clipped/and padded to obtain each image of size, $1024 \times 1024$ pixels. All images are stored in portable gray map (.pgm) format and are 8-bit gray level scale images with the dynamic intensity range as [0-255]. The image database was established by the Mammographic Image Analysis Society for research. The mammograms are arranged in pairs, with each pair representing a single patient's left and right breast. The study has used 10 images from every type of breast tissues in normal mammogram images.

\section{Methods}

\subsection{Thresholding}

In threshold-based segmentation, for a chosen threshold value, say $t$; the input image $I$, with the pixel intensity, say $i$ get transformed into a binary intensity image, with two ranges of intensities $i>t$ and $i \leq t$, which conventionally denote the foreground and background of the image, respectively. This study is required to determine the optimal threshold value that can ideally separate the foreground breast region and background. The following sections explain the principle 
of Isoentropic method, Kapur method, Kittler and Illingworth's method, Yen method, Ridler and Calvard's method and Otsu's method.

The computation of weighted sum, mean and variance for the extraction of background and foreground of an image is given in Table 1.

Table 1. Statistical measures for background and foreground of images

\begin{tabular}{|l|c|c|}
\hline \multicolumn{1}{|c|}{ Statistics Measures } & Background (B) & Foreground (F) \\
\hline Weighted sum of the & $\omega_{B}(k)=\sum_{i=0}^{k} p_{i}$ & $\omega_{F}(k)=1-\omega_{B}$ \\
\hline variances $(\omega)$ & $\mu_{B}(k)=\sum_{i=0}^{k} \frac{i p_{i}}{\omega_{B}}$ & $\mu_{F}(k)=\sum_{i=k+1}^{L-1} \frac{i p_{i}}{\omega_{F}}$ \\
\hline Variance $(\sigma)$ & $\sigma_{B}(k)^{2}=\sum_{i=0}^{k}\left(i-\mu_{B}\right)^{2} \frac{p_{i}}{\omega_{B}}$ & $\sigma_{F}(k)^{2}=\sum_{i=k+1}^{L-1}\left(i-\mu_{F}\right)^{2} \frac{p_{i}}{\omega_{F}}$ \\
\hline \multicolumn{2}{|l|}{ where, $p_{i}$-is the probability of occurrence of $i^{\text {th }}$ gray level } \\
\hline
\end{tabular}

The basic principles of the six threshold based segmentation methods selected for this research are explained in the following section.

\subsubsection{Isoentropic method}

This method sets an optimal threshold value $(\mathrm{k})$ that splits the grey-scale histogram into two parts of equal entropy, where entropy $E(j)$ is expressed as [17]:

$$
E(j)=\sum_{i=0}^{j} p_{i} \log _{2} \frac{1}{p_{i}}
$$

\subsubsection{Kapur's method}

Kapur's method [18], sometimes referred to as maximum entropy criterion $K(T)$ which is taken as a threshold that maximizes the sum of foreground $\left(\mathrm{E}_{\mathrm{F}}\right)$ and background entropy $\left(\mathrm{E}_{\mathrm{B}}\right)$ :

$$
K(T)=\underset{k \in\{0, \ldots, L-1\}}{\operatorname{argmax}}\left[\left(E_{B}+E_{F}\right)\right]
$$

where,

$$
\begin{aligned}
& E_{B}=\frac{1}{\omega_{B}} \sum_{i=0}^{j} p_{i} \log _{2} \frac{\omega_{B}}{p_{i}}, \quad \text { and } \\
& E_{F}=\frac{1}{\omega_{F}} \sum_{i=k+1}^{L-1} p_{i} \log _{2} \frac{\omega_{F}}{p_{i}}
\end{aligned}
$$




\subsubsection{Kittler-Illingworth method}

This method assumes that the gray-scale histogram can be approximated through a mixture of two Gaussian distributions, one for the foreground and the other for the background and sets the threshold $K(T)$, it minimizes the error between the original histogram and the mixture of the two approximating distributions [19]. The threshold function $\mathrm{K}(\mathrm{T})$ is expressed as $K(T)=1+2\left[p_{1}(T) \log \sigma_{B}^{2}(k)+p_{2}(T) \log \sigma_{F}^{2}(k)\right]-2\left[p_{1}(T) \log p_{1}(T)+p_{2}(T) \log p_{2}(T)\right]$

\subsubsection{Otsu's method}

Otsu's thresholding covers all possible thresholds and calculates the pixel values on each side of the threshold. This method selects a k-value that separates the foreground from the background based on the chosen threshold. It uses Intra-class variance, which is the weighted sum of the respective foreground and background variances [20].

$$
\sigma_{\text {within }}^{2}(T)=\omega_{B}(k) \sigma_{B}(k)^{2}+\omega_{F}(k) \sigma_{F}(k)^{2}
$$

\subsubsection{Ridler and Calvard's Method}

This method computes the initial threshold value $\mathrm{K}_{0}$ for the given image, which is the average of all pixel intensity values [21]. It divides the picture into two classes as foreground and background. Then it computes the mean of the foreground classes as $\mu_{f}$ and background as $\mu_{b} . K_{F}$ and $K_{B}$, which are the mean values of the foreground and background pixels respective. The improved threshold value is $K_{1}$ :

$$
K_{1}=\frac{K_{B}+K_{F}}{2}
$$

This method computes the threshold value using Eqn.(7). The new threshold is denoted as $K_{1}$ and the previous one as $K_{0}$. Finally, $K_{1}$ is taken as the threshold value when $K_{1}$ approaches close to $K_{0}$.

\subsubsection{Yen Method}

This technique is a variant of Kapur's method. The threshold is set at the value that maximizes the sum of correlated background and foreground. This method can be referred to as 'correlation' and also referred to as the maximum correlation criterion [22]. The correlation of foreground $\left(C_{F}\right)$ and background $\left(C_{B}\right)$ is denoted as follows:

$$
K(T)=\underset{k \in\{0, \ldots, L-1\}}{\operatorname{argmax}}\left[\left(C_{B}+C_{F}\right)\right]
$$


where,

$$
C_{B}=\log _{2}\left[\sum_{i=0}^{k}\left(\frac{\omega_{B}}{p_{i}}\right)^{2}\right] \quad C_{F}=\log _{2}\left[\sum_{j=k+1}^{L-1}\left(\frac{\omega_{F}}{p_{i}}\right)^{2}\right]
$$

\section{Results and Discussion}

In this research work, the efficiency of the six selected methods are analyzed with the Region Non-Uniformity (RNU). The computation of RNU is given in Eqn.10.

$$
R N U=\frac{\left|F G_{F}\right|}{\left|F G_{F}+B G_{B}\right|} \frac{\sigma_{F}^{2}}{\sigma^{2}}
$$

Where, the variance of the whole image is denoted as $\sigma^{2}$ and the variance of the foreground is denoted as $\sigma_{F}^{2}$. A well-segmented image has non-uniformity near zero. In the worst case, RNU becomes 1 . The RNU measure does not need the ground truth data to validate the accuracy of segmentation.

This study evaluates the performance of each of these threshold algorithms used to obtain the breast regions segmentation. The results of sample Dense-Glandular mammogram images for six thresholding methods are shown in Fig.1. 

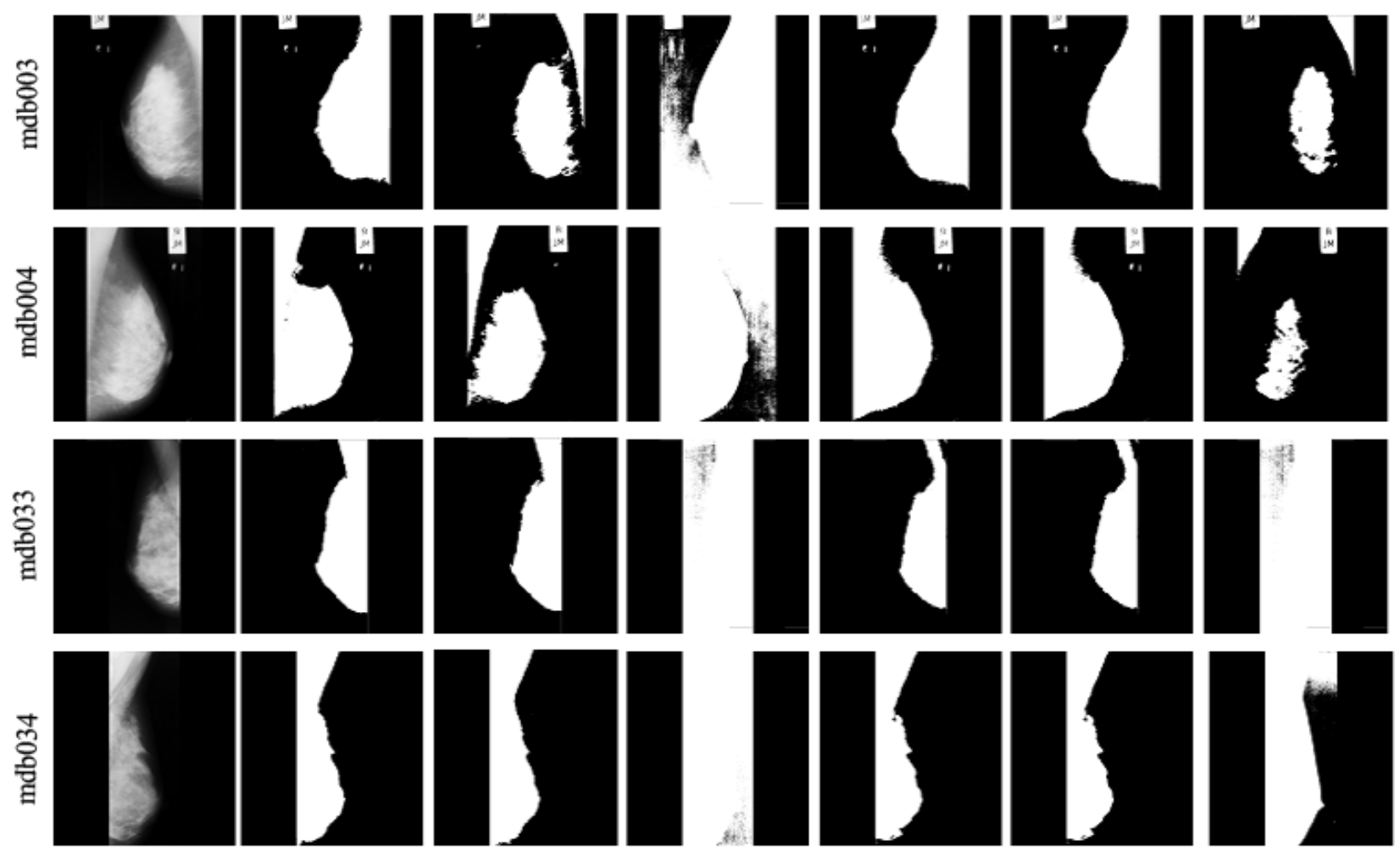

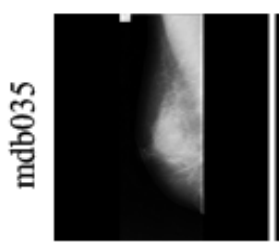

(a)

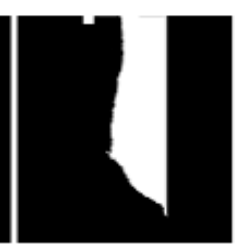

(b)

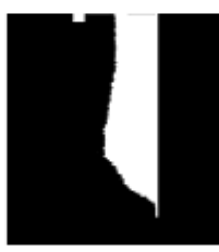

(c)

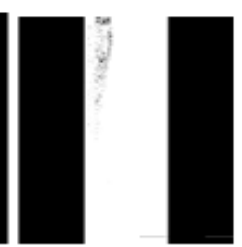

(d)

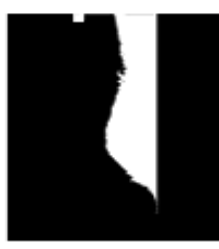

(e)

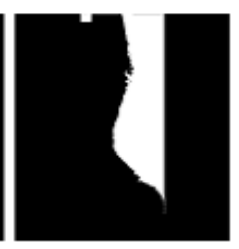

(f)

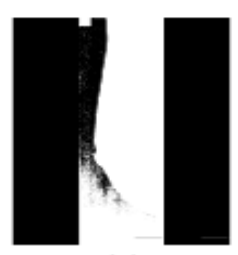

(g)

Fig.1. (a) Normal Dense-Glandular mammograms; Results of segmentation by (b) Isoentropic method, (c) Kapur method, (d) Kittler and Illingworth method, (e) Otsu method,

(f) Ridler and Calvard method and (g) Yen method. 


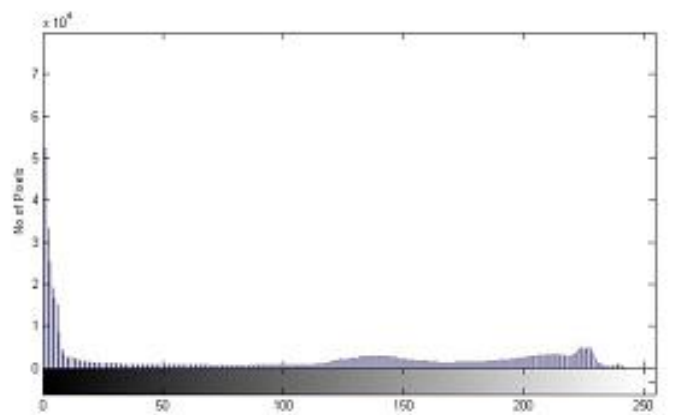

(a)

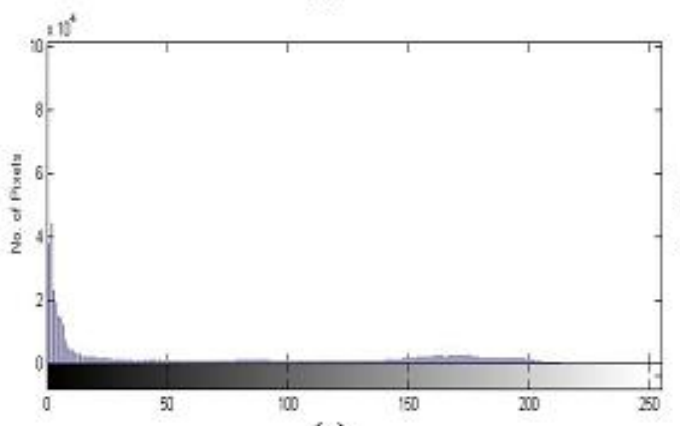

(c)

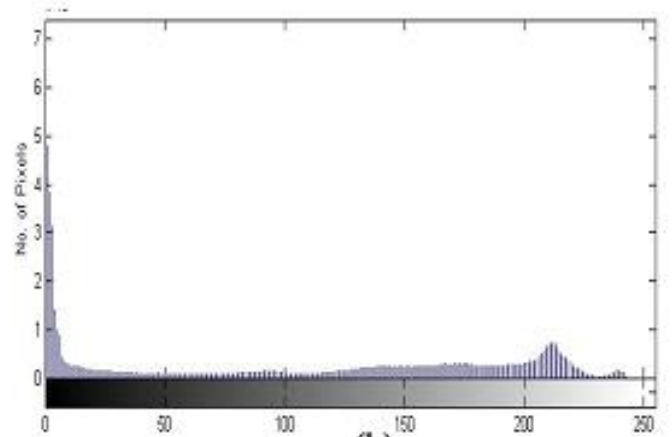

(b)

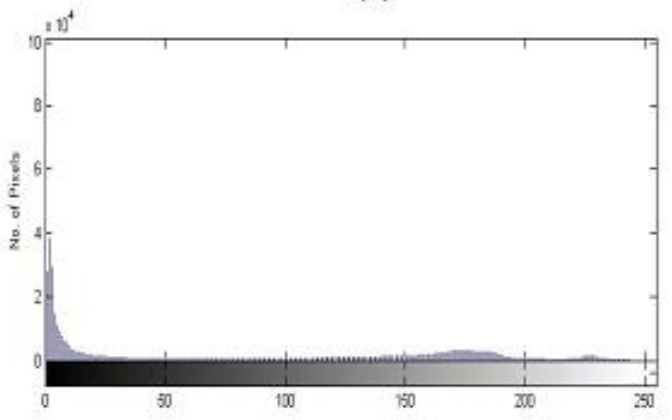

(d)

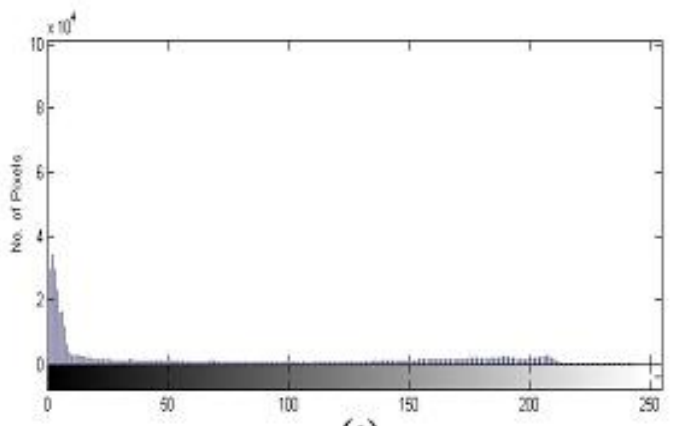

(e)

Fig.2. Histogram of Normal Dense-Glandular Mammogram images, (a) mdp003

(b) mdp004 (c) mdp033 (d) mdp034 and (e) mdp035

In order to depict the intensity distribution of the Normal Dense-Glandular mammogram input images, the histogram of these five input sample images is depicted in Fig.2.(a) to (e). It is apparent that the intensity of the maximum number of breast tissues is observed to be greater than 150 , that is the average intensity of the gray level

The observation of the experimental results vouch that Isoentropic, Kapur, Kittler \& Illingworth and Yen methods take the first-order probability distribution of the input image (i.e. histogram). Hence, these methods are proved to be invariant to any spatial redistribution of the original intensity values of the images. 
The Otsu and Ridler \& Calvard's methods compute the threshold based on the statistical mean of the background and foreground pixels of the images in the mini-MIAS dataset.

The RNU values obtained ten select on Normal Dense-Glandular mammograms for Isoentropic, Kapur, Kittler and Illingworth's, Otsu's, Ridler and Calvard's and Yen are recorded in Table 1. For each trial image-specific threshold was assigned as depicted in Table 2. It is observed that the Otsu's and Ridler \& Calvard's methods have produced almost the same results.

Table 2. The performance analysis of Threshold value (T) and RNU for Dense-glandular (D)

\begin{tabular}{|c|c|c|c|c|c|c|c|c|c|c|c|c|}
\hline \multirow{2}{*}{$\begin{array}{l}\text { Methods } \\
\text { Images }\end{array}$} & \multicolumn{2}{|c|}{ Isoentropic } & \multicolumn{2}{|c|}{ Kapur } & \multicolumn{2}{|c|}{$\begin{array}{l}\text { Kittler } \\
\text { and } \\
\text { Illingworth }\end{array}$} & \multicolumn{2}{|c|}{ Otsu } & \multicolumn{2}{|c|}{$\begin{array}{c}\text { Ridler } \\
\text { and } \\
\text { Calvard }\end{array}$} & \multicolumn{2}{|c|}{ Yen } \\
\hline & $T$ & $R N U$ & $T$ & $R N U$ & $T$ & $R N U$ & $T$ & $R N U$ & $T$ & $R N U$ & $T$ & $R N U$ \\
\hline mdb003 & 111 & 0.1565 & 150 & 0.0781 & 1 & 0.4819 & 90 & 0.1822 & 90 & 0.1822 & 193 & 0.019 \\
\hline mdb004 & 119 & 0.1009 & 154 & 0.1535 & 1 & 0.4457 & 91 & 0.1383 & 91 & 0.1383 & 205 & 0.0105 \\
\hline mdb033 & 32 & 0.7027 & 44 & 0.5954 & 1 & 0.6137 & 78 & 0.354 & 78 & 0.354 & 2 & 1.0654 \\
\hline mdb034 & 61 & 0.3263 & 48 & 0.9623 & 1 & 0.6127 & 83 & 0.2518 & 83 & 0.2518 & 6 & 0.9759 \\
\hline mdb035 & 40 & 0.6492 & 54 & 0.5912 & 1 & 0.613 & 85 & 0.2881 & 85 & 0.2881 & 4 & 1.1524 \\
\hline mdb036 & 61 & 0.4168 & 63 & 0.5894 & 1 & 0.6199 & 86 & 0.3028 & 86 & 0.3028 & 5 & 1.024 \\
\hline mdb040 & 98 & 0.4196 & 37 & 0.8212 & 1 & 0.8084 & 80 & 0.508 & 80 & 0.508 & 226 & 0.1383 \\
\hline mdb057 & 136 & 0.0824 & 173 & 0.1688 & 1 & 0.5343 & 79 & 0.1092 & 79 & 0.1092 & 158 & 0.0897 \\
\hline mdb062 & 75 & 0.1401 & 21 & 0.4964 & 3 & 0.9064 & 77 & 0.1357 & 78 & 0.1335 & 6 & 0.908 \\
\hline mdb064 & 123 & 0.1036 & 142 & 0.1301 & 1 & 0.6146 & 84 & 0.1796 & 84 & 0.1796 & 140 & 0.0769 \\
\hline Mean & & 0.3098 & & 0.4586 & & 0.6251 & & 0.2449 & & 0.2447 & & 0.546 \\
\hline
\end{tabular}

The purpose of this experiment was to validate the influence of the given threshold value on segmentation, measured as RNU. Fig.3 portrays the output images of the normal Fatty mammogram images chosen from the mini-MIAS dataset for the chosen methods. 


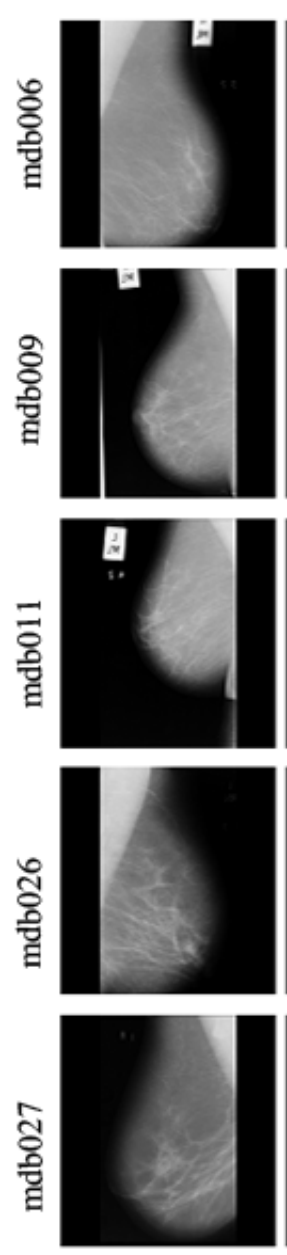

(a)
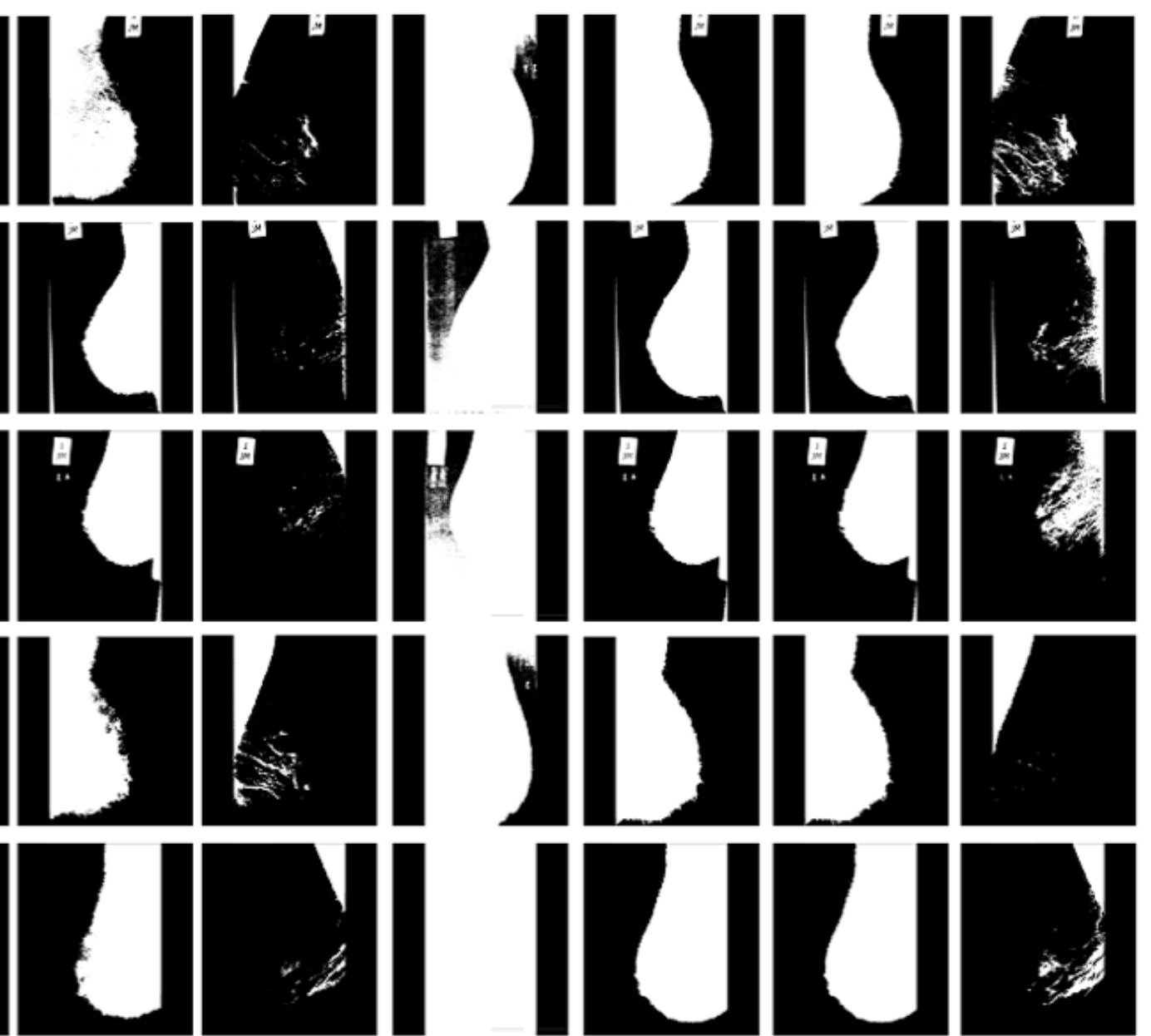

(b)
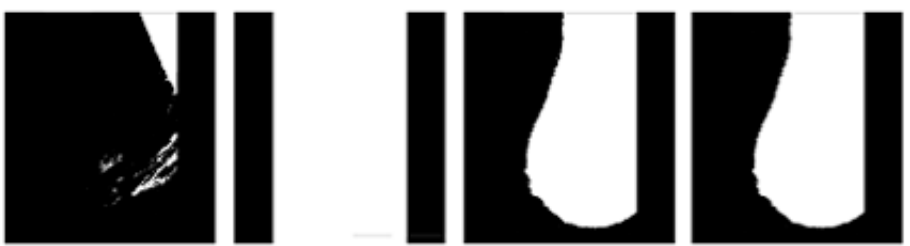

(e)

(f)

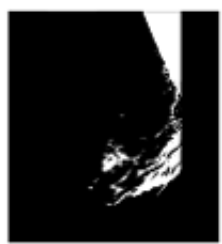

(d)

(g)

Fig.3. (a) Normal Fatty mammograms; Results of segmentation by (b) Isoentropic method, (c) Kapur method, (d) Kittler and Illingworth method, (e) Otsu method, (f) Ridler and Calvard method and $(\mathrm{g})$ Yen method. 


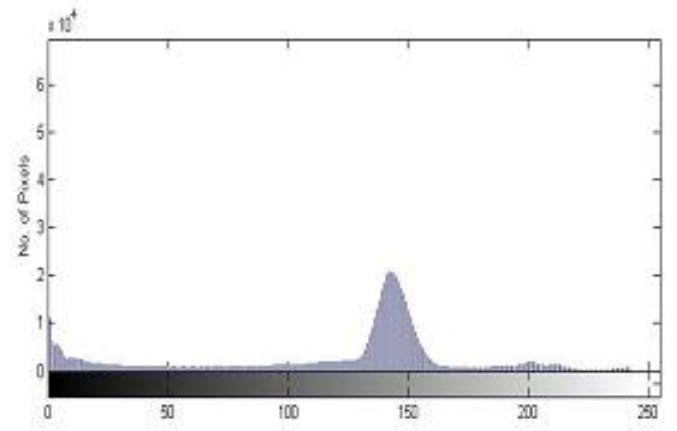

(a)

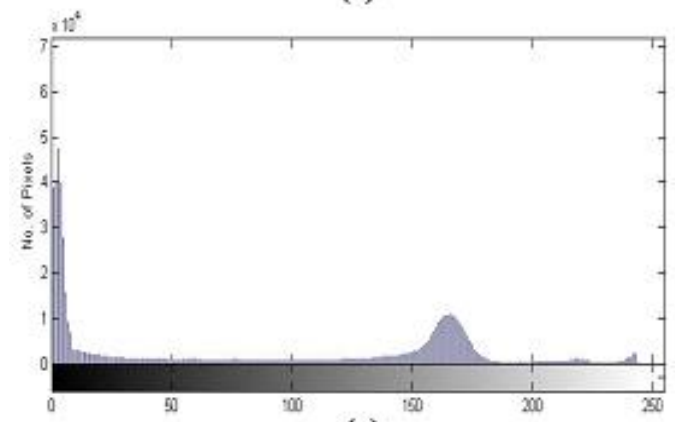

(c)

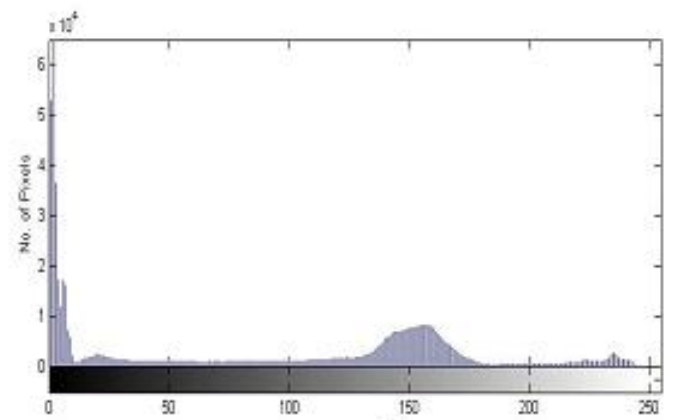

(b)

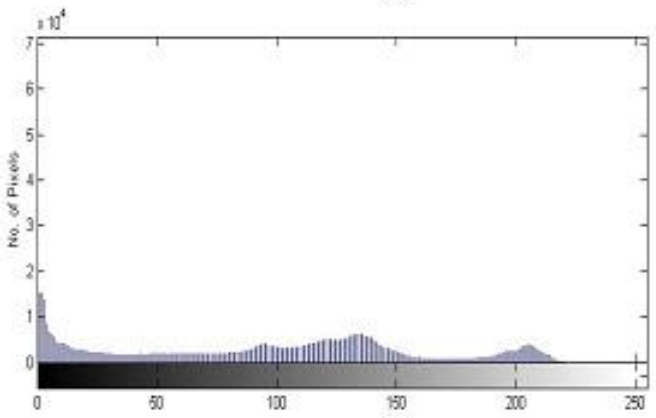

(d)

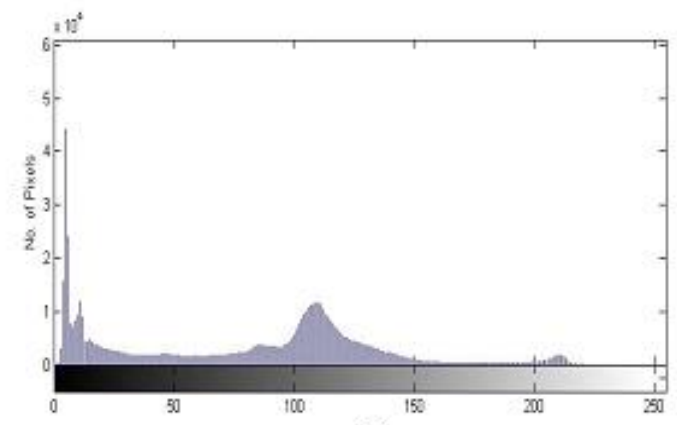

(e)

Fig. 4. Histogram of Normal Fatty Mammogram images, (a) mdp006, (b)mdp009 (c) mdp011 (d) mdp026 and (e) mdp027

The histogram of the respective five Fatty Mammogram images is shown in Fig.4.(a) to (e). The histogram of the five mammogram images shows that the maximum number of breast tissue regions of pixels distributed between the intensity values of 100 to 150. As observed from the results of those experimental methods on the normal fatty images, the accuracy of segmentation by Kapur, Kittler \& Illingworth, Otsu, Ridler \& Calvard's and Yen methods were not good. The Isoentropic method is proved to segment better than its counterparts. 
Table 3 shows the threshold value (T) and RNU obtained algorithms for a normal mammogram image for fatty tissues. The RNU of the isoentropic system is observed to be close to zero. This thresholding method showed the best results for fatty mammogram images.

Table 3. The performance analysis of Threshold value $(\mathrm{T})$ and RNU for Fatty (F)

\begin{tabular}{|c|c|c|c|c|c|c|c|c|c|c|c|c|}
\hline \multirow{2}{*}{ Images } & \multicolumn{2}{|c|}{ Isoentropic } & \multicolumn{2}{|c|}{ Kapur } & \multicolumn{2}{|c|}{$\begin{array}{c}\text { Kittler } \\
\text { and } \\
\text { Illingworth }\end{array}$} & \multicolumn{2}{|c|}{ Otsu } & \multicolumn{2}{|c|}{$\begin{array}{c}\text { Ridler } \\
\text { and } \\
\text { Calvard }\end{array}$} & \multicolumn{2}{|c|}{ Yen } \\
\hline & $T$ & $R N U$ & $T$ & $R N U$ & $T$ & $R N U$ & $T$ & $R N U$ & $T$ & $R N U$ & $T$ & $R N U$ \\
\hline mdb006 & 135 & 0.0548 & 162 & 0.0838 & 1 & 0.4193 & 75 & 0.072 & 75 & 0.072 & 153 & 0.113 \\
\hline mdb009 & 99 & 0.1234 & 173 & 0.0988 & 4 & 0.4722 & 79 & 0.1454 & 80 & 0.144 & 162 & 0.1487 \\
\hline mdb011 & 74 & 1241 & 180 & 0.0891 & 1 & 0.4268 & 82 & 0.1271 & 83 & 0.1252 & 165 & 0.0827 \\
\hline mdb026 & 95 & 0.1227 & 147 & 0.1759 & 1 & 0.4342 & 71 & 0.2057 & 71 & 0.2057 & 170 & 0.0269 \\
\hline mdb027 & 85 & 0.1552 & 145 & 0.2233 & 1 & 0.3652 & 61 & 0.1713 & 61 & 0.1713 & 134 & 0.2379 \\
\hline mdb060 & 80 & 0.2602 & 127 & 0.2707 & 4 & 0.7707 & 55 & 0.2886 & 55 & 0.2886 & 0 & 0.7745 \\
\hline mdb070 & 90 & 0.08 & 107 & 0.0562 & 1 & 0.3653 & 75 & 0.1007 & 75 & 0.1007 & 163 & 0.0113 \\
\hline mdb076 & 117 & 0.1176 & 155 & 0.1703 & 1 & 0.5 & 79 & 0.1719 & 80 & 0.1708 & 145 & 0.1376 \\
\hline mdb077 & 126 & 0.0772 & 158 & 0.1126 & 1 & 0.4459 & 73 & 0.1011 & 74 & 0.1002 & 150 & 0.1295 \\
\hline mdb078 & 118 & 0.0822 & 166 & 0.0791 & 1 & 0.5188 & 73 & 0.1223 & 73 & 0.1223 & 149 & 0.0904 \\
\hline Mean & & 0.1197 & & 0.1359 & & 0.4718 & & 0.1506 & & 0.1501 & & 0.1752 \\
\hline
\end{tabular}

This analysis reveals the impact of threshold on the thresholding algorithm for breast tissue segmentation. The results of sample Fatty-Glandular mammogram images for six thresholding methods are shown in Fig.5. 

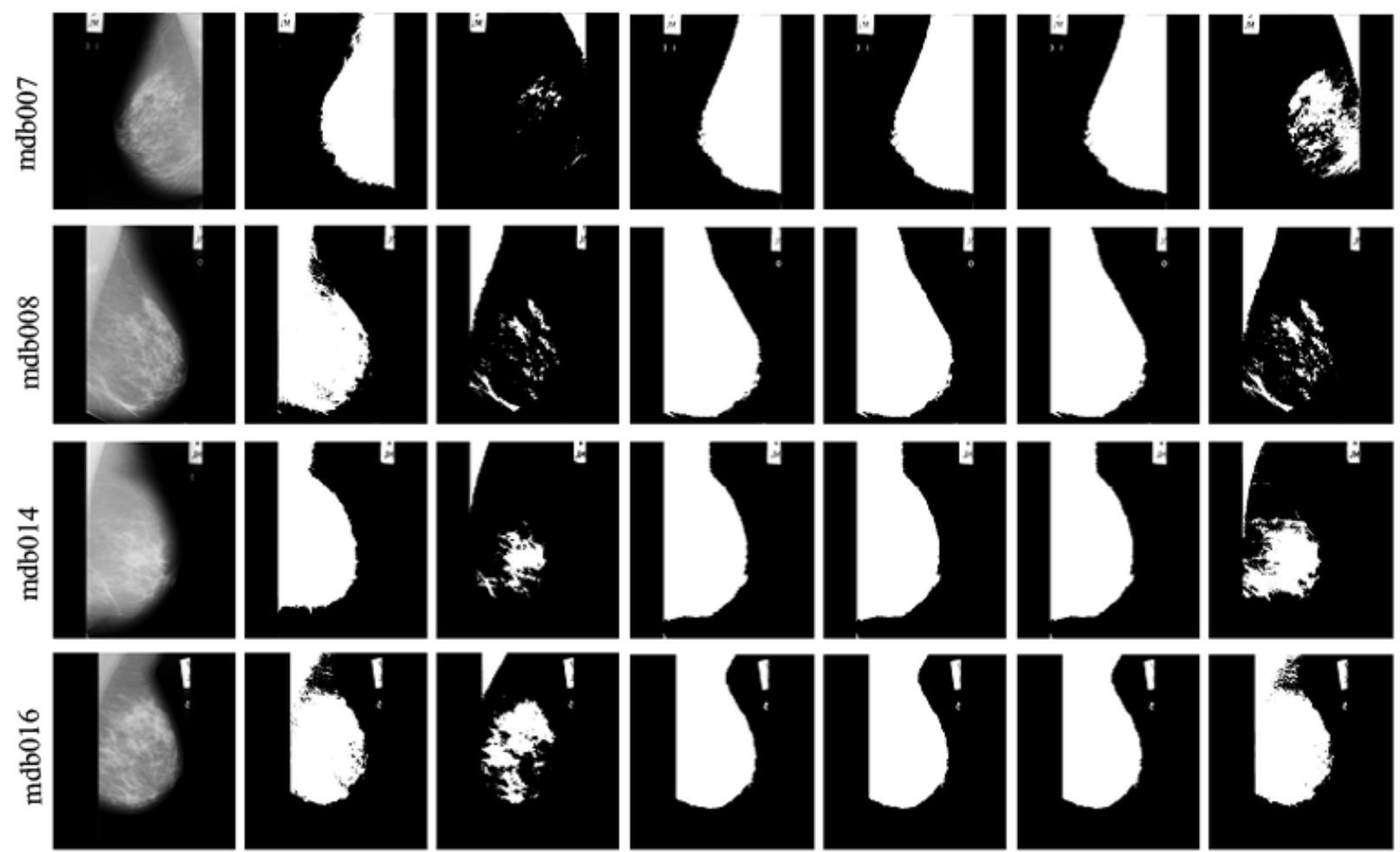

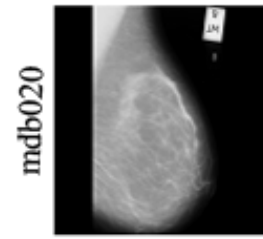

(a)

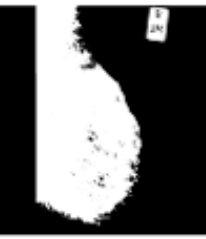

(b)

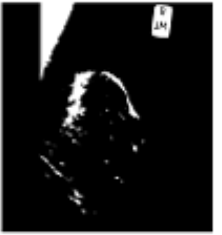

(c)

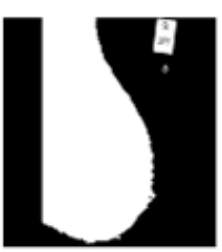

(d)

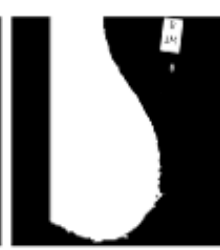

(e)

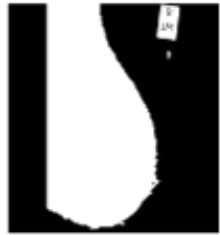

(f)

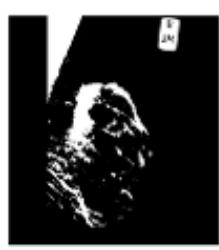

(g)

Fig. 5. (a) Normal Fatty-Glandular mammograms; Results of segmentation by (b) Isoentropic method, (c) Kapur method, (d) Kittler and Illingworth method, (e) Otsu method, (f) Ridler and Calvard method and (g) Yen method. 


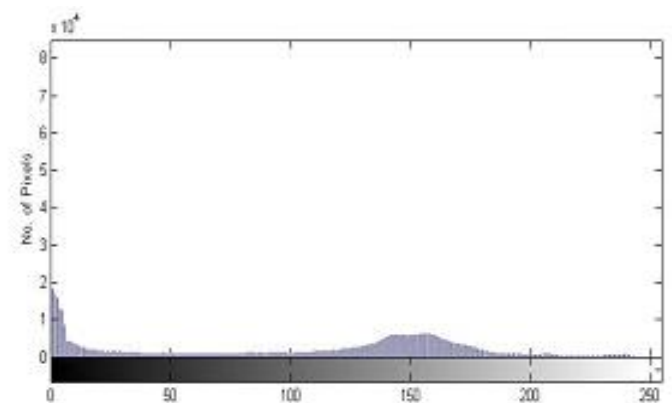

(a)

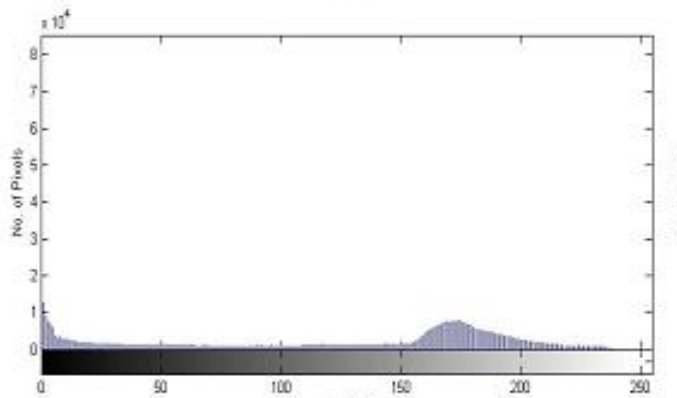

(c)

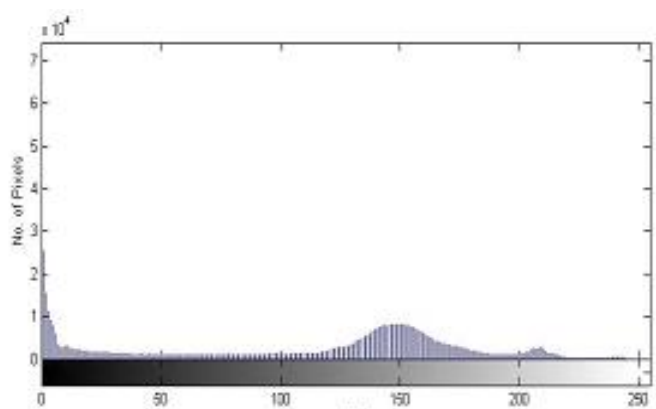

(b)

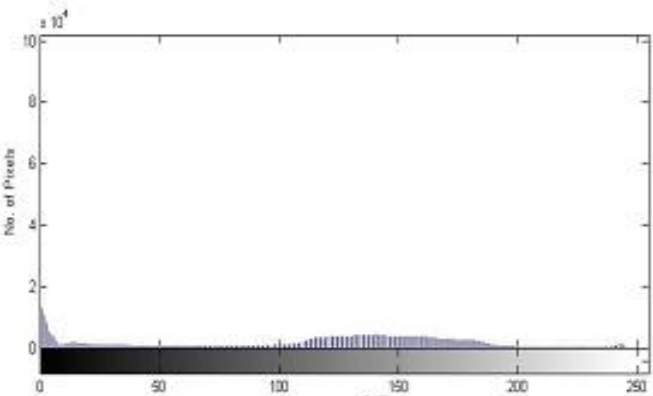

(d)

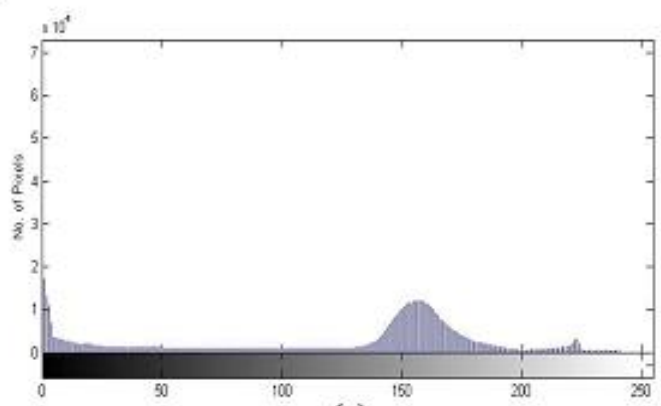

(e)

Fig. 6. Histogram of Fatty-Glandular Mammogram images, (a) mdp007 (b) mdp008 (c) mdp014 (d) mdp016 and (e) mdp020

The histogram of the respective five Fatty-Glandular Mammogram images is shown in Fig.6 (a) to (e). The histogram of the five mammogram images shows that the maximum number of breast tissue regions of pixels distributed between the intensity values of 100 to 200 . The thresholding methods, Kapur, Kittler \& Illingworth's, Otsu, Ridler \& Calvard's and Yen methods cannot give well segmented images for Fatty Mammogram. Therefore Isoentropic method has given well segmented images for Fatty mammograms.

Table 4 shows the threshold value (T) and RNU value obtained by thresholding algorithms for fatty tissue in a normal mammogram image. The Yen method's RNU is close to zero. For FattyGlandular mammogram images, this thresholding approach generated the best results. 
Table 4. The performance analysis of Threshold value (T) and RNU for Fatty-Glandular (G)

\begin{tabular}{|c|c|c|c|c|c|c|c|c|c|c|c|c|}
\hline \multirow{2}{*}{ Images } & \multicolumn{2}{|c|}{ Isoentropic } & \multicolumn{2}{|c|}{ Kapur } & \multicolumn{2}{|c|}{$\begin{array}{c}\text { Kittler } \\
\text { and } \\
\text { Illingworth }\end{array}$} & \multicolumn{2}{|c|}{ Otsu } & \multicolumn{2}{|c|}{$\begin{array}{c}\text { Ridler } \\
\text { and } \\
\text { Calvard }\end{array}$} & \multicolumn{2}{|c|}{ Yen } \\
\hline & $T$ & $R N U$ & $T$ & $R N U$ & $T$ & $R N U$ & $T$ & $R N U$ & $T$ & $R N U$ & $T$ & $R N U$ \\
\hline $\mathrm{mdb} 007$ & 116 & 0.0802 & 183 & 0.0637 & 1 & 0.5158 & 76 & 0.126 & 76 & 0.126 & 155 & 0.0679 \\
\hline $\mathrm{mdb} 008$ & 129 & 0.0666 & 173 & 0.0566 & 1 & 0.4505 & 78 & 0.0994 & 78 & 0.0994 & 168 & 0.0567 \\
\hline $\mathrm{mdb} 014$ & 139 & 0.0425 & 175 & 0.1925 & 1 & 0.5195 & 88 & 0.0944 & 88 & 0.0944 & 180 & 0.0283 \\
\hline mdb016 & 122 & 0.0994 & 152 & 0.0728 & 1 & 0.6214 & 73 & 0.1443 & 74 & 0.1432 & 117 & 0.105 \\
\hline $\mathrm{mdb} 020$ & 144 & 0.045 & 181 & 0.0495 & 1 & 0.4423 & 82 & 0.0732 & 82 & 0.0732 & 171 & 0.0617 \\
\hline $\mathrm{mdb} 022$ & 89 & 0.1382 & 88 & 0.1403 & 1 & 0.5085 & 81 & 0.1558 & 81 & 0.1558 & 158 & 0.0455 \\
\hline $\mathrm{mdb} 024$ & 68 & 0.1329 & 194 & 0.1819 & 1 & 0.3652 & 82 & 0.1001 & 83 & 0.098 & 172 & 0.0173 \\
\hline $\mathrm{mdb} 029$ & 104 & 0.0625 & 73 & 0.1006 & 2 & 0.403 & 97 & 0.0696 & 98 & 0.0685 & 203 & 0.0053 \\
\hline $\mathrm{mdb} 031$ & 83 & 0.1591 & 123 & 0.0829 & 2 & 0.3829 & 81 & 0.1635 & 81 & 0.1635 & 184 & 0.0111 \\
\hline $\mathrm{mdb} 041$ & 103 & 0.1684 & 152 & 0.1887 & 1 & 0.5521 & 78 & 0.2095 & 78 & 0.2095 & 141 & 0.1204 \\
\hline Mean & & 0.0994 & & 0.1129 & & 0.4761 & & 0.1235 & & 0.1231 & & 0.0519 \\
\hline
\end{tabular}

The segmented methods selected for the comparative analysis were executed in a MATLAB R2013a on a PC with Intel(R) Core(TM) $2.20 \mathrm{GHz}$ processor and 4 GB RAM. The obtained computation time of six thresholding methods for Dense-Glandular, Fatty and FattyGlandular are shown in Tables 5,6 and 7. The results reveal that the execution time of Kapur is recorded Dense-Glandular as 0.0076, Fatty as 0.0073 and Fatty-Glandular as 0.0075, which is the minimum among the obtained results than the remaining thresholding methods. 
Table 5. The Computation Time of thresholding methods for Dense-glandular (D)

\begin{tabular}{|c|c|c|c|c|c|c|}
\hline \multirow{2}{*}{ Methods } & \multicolumn{7}{|c|}{ Computation Time (Sec) } & \multicolumn{2}{c|}{$\begin{array}{c}\text { Ridler } \\
\text { and } \\
\text { Images }\end{array}$} & Isoentropic & Kapur & $\begin{array}{c}\text { Kittler } \\
\text { and } \\
\text { Illingworth }\end{array}$ & Yen \\
\hline mdb003 & 0.0285 & 0.0071 & 0.0199 & 0.0214 & 0.0342 & 0.0082 \\
\hline mdb004 & 0.0283 & 0.0103 & 0.0183 & 0.0121 & 0.0328 & 0.0079 \\
\hline mdb033 & 0.0281 & 0.0071 & 0.0179 & 0.0119 & 0.0301 & 0.0076 \\
\hline mdb034 & 0.0281 & 0.0072 & 0.0179 & 0.0122 & 0.0339 & 0.0076 \\
\hline mdb035 & 0.0294 & 0.0073 & 0.0186 & 0.0124 & 0.0324 & 0.0076 \\
\hline mdb036 & 0.0305 & 0.0078 & 0.0181 & 0.012 & 0.0352 & 0.0079 \\
\hline mdb040 & 0.0393 & 0.0073 & 0.0212 & 0.0124 & 0.0318 & 0.0077 \\
\hline mdb057 & 0.0304 & 0.0076 & 0.0183 & 0.0123 & 0.0399 & 0.0112 \\
\hline mdb062 & 0.0302 & 0.0074 & 0.0181 & 0.012 & 0.0314 & 0.0115 \\
\hline mdb064 & 0.0292 & 0.0073 & 0.0185 & 0.0135 & 0.0334 & 0.0111 \\
\hline Mean & 0.0302 & $\mathbf{0 . 0 0 7 6}$ & 0.0187 & 0.0132 & 0.0335 & 0.0088 \\
\hline
\end{tabular}

Table 6. The Computation Time of thresholding methods for Fatty (F)

\begin{tabular}{|c|c|c|c|c|c|c|}
\hline \multirow[t]{2}{*}{ Methods } & \multicolumn{6}{|c|}{ Computation Time (Sec) } \\
\hline & Isoentropic & Kapur & $\begin{array}{c}\text { Kittler } \\
\text { and } \\
\text { Illingworth }\end{array}$ & Otsu & $\begin{array}{c}\text { Ridler } \\
\text { and } \\
\text { Calvard }\end{array}$ & Yen \\
\hline mdb006 & 0.0303 & 0.0082 & 0.0193 & 0.0122 & 0.0332 & 0.0075 \\
\hline mdb009 & 0.0296 & 0.0071 & 0.0184 & 0.0118 & 0.033 & 0.0087 \\
\hline mdb011 & 0.029 & 0.0072 & 0.0181 & 0.0125 & 0.0327 & 0.0077 \\
\hline mdb026 & 0.0353 & 0.0072 & 0.018 & 0.0121 & 0.031 & 0.0088 \\
\hline mdb027 & 0.0294 & 0.0071 & 0.0179 & 0.0158 & 0.0336 & 0.0075 \\
\hline mdb060 & 0.0288 & 0.0072 & 0.0178 & 0.012 & 0.0307 & 0.0079 \\
\hline mdb070 & 0.0311 & 0.0071 & 0.0181 & 0.012 & 0.031 & 0.0076 \\
\hline mdb076 & 0.0293 & 0.0077 & 0.018 & 0.0122 & 0.0329 & 0.0075 \\
\hline mdb077 & 0.0288 & 0.0072 & 0.0176 & 0.0122 & 0.0327 & 0.0078 \\
\hline
\end{tabular}




\begin{tabular}{|c|c|c|c|c|c|c|}
\hline mdb078 & 0.0285 & 0.0073 & 0.0177 & 0.012 & 0.0326 & 0.0075 \\
\hline Mean & 0.0301 & $\mathbf{0 . 0 0 7 3}$ & 0.0181 & 0.0125 & 0.0323 & 0.0079 \\
\hline
\end{tabular}

Table 7. The Computation Time of thresholding methods for Fatty-glandular (G)

\begin{tabular}{|c|c|c|c|c|c|c|}
\hline \multirow{2}{*}{ Methods } & \multicolumn{7}{|c|}{ Computation Time (Sec) } \\
\cline { 2 - 7 } Images & Isoentropic & Kapur & $\begin{array}{c}\text { Kittler } \\
\text { and } \\
\text { Illingworth }\end{array}$ & Otsu & $\begin{array}{c}\text { Ridler } \\
\text { and } \\
\text { Calvard }\end{array}$ & Yen \\
\hline mdb006 & 0.0297 & 0.0071 & 0.0214 & 0.0121 & 0.0325 & 0.0077 \\
\hline mdb009 & 0.0295 & 0.0071 & 0.0184 & 0.0124 & 0.0325 & 0.0076 \\
\hline mdb011 & 0.0299 & 0.0094 & 0.0198 & 0.0119 & 0.0353 & 0.0076 \\
\hline mdb026 & 0.0285 & 0.0072 & 0.0189 & 0.0119 & 0.0922 & 0.0076 \\
\hline mdb027 & 0.0304 & 0.0074 & 0.0181 & 0.0118 & 0.0323 & 0.0076 \\
\hline mdb060 & 0.0319 & 0.0075 & 0.0179 & 0.0118 & 0.0315 & 0.0075 \\
\hline mdb070 & 0.0306 & 0.0072 & 0.0177 & 0.0119 & 0.0327 & 0.0081 \\
\hline mdb076 & 0.0286 & 0.0072 & 0.0177 & 0.0118 & 0.0327 & 0.0076 \\
\hline mdb077 & 0.0302 & 0.0074 & 0.0179 & 0.0121 & 0.0321 & 0.0075 \\
\hline mdb078 & 0.0298 & 0.0074 & 0.0182 & 0.0122 & 0.0326 & 0.0111 \\
\hline Mean & 0.0299 & $\mathbf{0 . 0 0 7 5}$ & 0.0186 & 0.0119 & 0.0386 & 0.0081 \\
\hline
\end{tabular}

\section{Conclusions}

This research work was designed to analyse the association between the selection of threshold value for threshold-based segmentation methods, with reference to Isoentropic, Kapur, Kittler \& Illingworth's, Otsu's, Ridler \& Calvard's, and Yen mammogram images of mini-MIAS dataset. The performance was tested on the different types of mammogram images are DenseGlandular, Fatty and Fatty-Glandular. The effectiveness of segmentation was assessed using RNU and computation time. This study confirms that Ridler and Calvard's method gives the best results for Dense-Glandular segmentation, the Isoentropic method shows better results on Fatty, and the Yen method works well on the Fatty-Glandular normal mammogram images. It is further vouched that the Kapur method is proved to be computationally simple. 


\section{References}

1. Michaelson, J., Satija, S., Moore, R., Weber, G., Halpern, E., Garland, A., Puri, D. and Kopans, D.B., "The pattern of breast cancer screening utilization and its consequences. Cancer", 94(1), 2002, pp.37-43.

2. Chen-Chung Liu, Chung-Yen Tsai, Jui Liu, Chun-Yuan Yu, Shyr-Shen Yu, “A pectoral muscle segmentation algorithm for digital mammograms using Otsu thresholding and multiple regression analysis", Computers and Mathematics with Applications, 64, 2012, pp. 1100-1107

3. Mustra, M., Grgic, M. and Rangayyan, R.M., 2016. Review of recent advances in segmentation of the breast boundary and the pectoral muscle in mammograms. Medical \& biological engineering \& computing, 54(7), pp.1003-1024.

4. Angelov, Plamen, Pouria Sadeghi-Tehran, and Ramin Ramezani. "An approach to automatic real-time novelty detection, object identification, and tracking in video streams based on recursive density estimation and evolving Takagi-Sugeno fuzzy systems." International Journal of Intelligent Systems, 26(3), 2011, pp.189-205.

5. R.C. Gonzalez, R.E. Woods, “Digital Image Processing,” Pearson Education, Inc., Publication, 2009.

6. S.S. Al-amri, N.V. Kalyankar and S.D. Khamitkar, "Image Segmentation using threshold techniques," Journal of Computing, 2, 2010, pp.83-86.

7. Ergin, Semih, İdil Işıklı Esener, and Tolga Yüksel. "A genuine GLCM-based feature extraction for breast tissue classification on mammograms." International Journal of Intelligent Systems and Applications in Engineering, 2016, 124-129.

8. Palkar, Pravin, and Pankaj Agrawal. "A technique to extract statistical parameters of digital mammogram to detect breast cancer." Int. J. Adv. Res. Sci. Eng. Technol, 3(12), 2016, pp.30333038.

9. Ibrahim, Naglaa S. Ali, Naglaa F. Soliman, Mahmoud Abdallah, and Fathi E. Abd El-Samie. "An algorithm for pre-processing and segmentation of mammogram images." In $201611^{\text {th }}$ International Conference on Computer Engineering \& Systems (ICCES), pp. 187-190. IEEE, 2016.

10. Qayyum, Abdul, and A. Basit. "Automatic breast segmentation and cancer detection via SVM in mammograms." In 2016 International conference on emerging technologies (ICET), pp. 1-6. IEEE, 2016. 
11. Salama MS, Eltrass AS, Elkamchouchi HM (2018) An improved approach for computer-aided diagnosis of breast cancer in digital mammography. In: 2018 IEEE international symposium onmedical measurements and applications (MeMeA), 2018. IEEE, pp 1-5

12. ESENER, İDİL IŞIKLI, Semih Ergin, and Tolga Yüksel. "A novel multistage system for the detection and removal of pectoral muscles in mammograms." Turkish Journal of Electrical Engineering \& Computer Sciences, 26 (1)2018, pp.35-49.

13. Ancy, C. A., and Lekha S. Nair. "Tumour classification in graph-cut segmented mammograms using GLCM features-fed SVM." In Intelligent engineering informatics, pp. 197-208. Springer, Singapore, 2018.

14. Bajaj, Varun, Mayank Pawar, Vinod Kumar Meena, Mukesh Kumar, Abdulkadir Sengur, and Yanhui Guo. "Computer-aided diagnosis of breast cancer using bi-dimensional empirical mode decomposition." Neural Computing and Applications 31 (8), 2019, pp.3307-3315.

15. Selvathi, D., and A. Aarthy Poornila. "Deep learning techniques for breast cancer detection using medical image analysis." In Biologically rationalized computing techniques for image processing applications, pp. 159-186. Springer, Cham, 2018.

16. MIAS database http://peipa.essex.ac.uk/info/mias.html

17. Bianconi, Francesco, Antonio Fernández, Elena González, and Fernando Ribas. "Texture classification through combination of sequential colour texture classifiers." In Iberoamerican Congress on Pattern Recognition, pp. 231-240. Springer, Berlin, Heidelberg, 2007.

18. Kapur, Jagat Narain, Prasanna K. Sahoo, and Andrew KC Wong. "A new method for graylevel picture thresholding using the entropy of the histogram." Computer vision, graphics, and image processing, 29(3), 1985, pp.273-285.

19. Kittler, Josef, and John Illingworth. "Minimum error thresholding." Pattern recognition 19, 1, 1986, pp.41-47.

20. Otsu, Nobuyuki. "A threshold selection method from gray-level histograms." IEEE transactions on systems, man, and cybernetics, 9(1), 1979, pp.62-66.

21.T. Ridler and S. Calvard, "Picture Thresholding using and Iterative Selection Method," IEEE Transactions of systems, Man and Cybernetics (SMC), 8(8), 1978, pp.630-632.

22. Yen, Jui-Cheng, Fu-Juay Chang, and Shyang Chang. "A new criterion for automatic multilevel thresholding." IEEE Transactions on Image Processing, 4(3), 1995, pp.370-378. 